\title{
Are we there yet? The never-ending quest for an Epstein-Barr virus vaccine
}

\author{
Sandhya Sharma and Rayne H. Rouce \\ Center for Cell and Gene Therapy, Baylor College of Medicine, Houston Methodist Hospital and Texas Children's Hospital, Houston, Texas, USA. Texas Children's Cancer and Hematology Centers, Baylor College \\ of Medicine, Houston, Texas, USA.
}

\begin{abstract}
The Epstein-Barr virus (EBV) is estimated to infect a large part of the population and is associated with a variety of human tumors; therefore, EBV is an important target for vaccine development. In this issue of the $J C I$, Rühl et al. developed a promising heterologous prime-boost vaccination strategy for EBV-associated malignancies and symptomatic primary infection. The authors show that two prime-boost regimens, using either dendritic cells or an adenovirus approach targeting nuclear antigen EBNA1 followed by a modified vaccinia virus Ankara (MVA) booster, induced significant T cellmediated, EBV-specific immune control and Ab production. These findings suggest that administration of heterologous prime-boost vaccinations targeting EBNA1 may result in potent $\mathrm{CD4}^{+}$and $\mathrm{CD} 8^{+} \mathrm{T}$ cell-mediated EBV immune control and may be a promising clinical approach.
\end{abstract}

\section{EBV: a prevalent cancer-associated virus}

Epstein-Barr virus (EBV) is a primary cause of infectious mononucleosis (IM) and is globally associated with various malignancies, most notably post-transplantation lymphoproliferative disease (PTLD), de novo Hodgkin/non-Hodgkin lymphoma (HL/NHL), and nasopharyngeal carcinoma (NPC) (1). Despite the development of preventive vaccines against other malignant viruses (2-5), and notwithstanding substantial efforts undertaken by a number of groups (6-8), an effective vaccine against EBV infection and malignancy prevention has yet to be developed. Some of the unique challenges facing the development of an EBV vaccine are: (a) the scarcity of available animal models for preclinical testing of EBV infection; (b) the differences in EBV life-cycle stages (lytic vs. latent cycles), which correlate with unique antigen expression and a varied immune response against the virus; (c) the absence of suitable biomarkers that predict the development of EBV-positive malignancies; and (d) the lengthy time period between EBV infection and tumor development $(9,10)$. In this issue, Rühl et al. show that a heterologous prime-boost vaccination strategy against nuclear antigen EBNA1 (E1) is able to protect against the development of cancer and serve as an effective therapy in two models of EBVassociated lymphoma (11).

\section{How far have we come?}

EBV infection is primarily controlled by a delicate balance of $\mathrm{B}$ and $\mathrm{T}$ cells. Outgrowth of EBV-infected B cells is a direct consequence of inadequate EBV-specific cytotoxic T lymphocytes, hence the higher incidence of EBV-associated malignancy in immunocompromised hosts (12). While no vaccine is currently available for the disease, adoptive transfer of EBV-specific $\mathrm{T}$ lymphocytes that recognize EBV antigens have emerged as a promising

- Related Article: p. 2071

Conflict of interest: The authors have declared that no conflict of interest exists.

Copyright: @ 2019, American Society for Clinical Investigation.

Reference information: / Clin Invest. 2019;129(5):1836-1838. https://doi.org/10.1172/JCI128370.

therapeutic option. These ex vivo-manufactured donor $\mathrm{T}$ cells and patient-derived EBV-specific $T$ cells have eradicated disease in patients with refractory $\mathrm{EBV}^{+}$ polymorphic and monomorphic PTLD (13-15). Thus, the role of $\mathrm{T}$ cells in controlling EBV in immunocompetent hosts and in eradicating EBV in immunocompromised hosts following ex vivo antigen-specific priming is clear and encourages the development and design of EBV vaccines. The quest for an EBV-directed vaccine has proven quite challenging, in large part because of the lack of preclinical models for vaccine development. Rühl and colleagues used two unique models of $\mathrm{T}$ and $\mathrm{B}$ cell lymphoma in mice with a C57BL/6 background to evaluate a heterologous prime-boost vaccination strategy aimed at preventing the development of and treating E1-expressing tumors.

Human trials of prophylactic EBV vaccines with the ability to induce neutralizing Abs in seronegative subjects against the EBV glycoprotein gp350 $(6-8,16)$ have shown clinical promise. These vaccines reduced the incidence of IM, but not EBV infection, as vaccinated individuals developed asymptomatic seroconversion (6, 7). Alternative approaches to prophylactic vaccination against EBV have included attempts to induce a $\mathrm{T}$ cell immune response against the EBV-latent cycle protein EBNA3a (17). Similar to the former approach, the EBNA3a study demonstrated vaccination-induced EBNA3a-specific $T$ cells, and in this study there was a reduced incidence of IM infection, though in a small sample size; however, the EBNA3abased vaccine did not protect subjects from EBV infection (17). Approaches aimed at boosting the adaptive immune system to prevent the development of EBV-associated malignancies have also been explored over the years. Therapeutic EBV vaccine strategies have targeted the type 2 latency proteins EBNA1, LMP1, and LMP2, which comprise the signature expression pro- 
file of HL and NPC. Vaccine strategies for patients with NPC have included autologous dendritic cells pulsed with LMP2 epitope peptides (18) and modified vaccinia Ankara virus (MVA) expressing the EBVlatent proteins EBNA1 and LMP2 $(19,20)$. In the dendritic cell-based approach, vaccination increased LMP2-specific CD8 ${ }^{+} \mathrm{T}$ cells followed by partial clinical responses (18). The MVA approach enhanced peripheral blood $\mathrm{CD}^{+}$and $\mathrm{CD}^{+} \mathrm{T}$ cell responses against EBNA1 and LMP2 $(19,20)$. An alternative approach involved vaccinating NPC patients with autologous dendritic cells that had been transduced with adenovirus-expressing LMP1 and LMP2 (21). Although no increase in LMP1- and/ or LMP2-specific T cells was observed, 3 of the 16 patients had clinical responses. One common feature of these early vaccination studies is the resultant enhancement in $\mathrm{CD}^{+}$and $\mathrm{CD}^{+} \mathrm{T}$ cell responses specific to the vaccine target antigen, leading to decreased tumor burden in some cases. Nevertheless, the overall clinical benefit remained limited. Therefore, despite the promising results of these trials, there is an obvious need for both prophylactic and therapeutic EBV vaccines that enhance adaptive immunity. Rühl et al. now present an innovative EBV vaccine concept aimed at inducing adaptive cellular immunity via cross-presentation of the EBV-latent gene EBNA1, thereby inducing enhanced immune responses of both $\mathrm{CD}^{+}$and $\mathrm{CD}^{+}$ T cells (11). Considering that EBV proteinencoding recombinant viral vectors have been safely evaluated in the clinical setting in patients with NPC $(19,20)$, and given the recent success of a prime-boost combination vaccine strategy with adenovirus and vaccinia virus in clinical trials targeting malaria (22), the prime-boost therapeutic EBV vaccination strategy described by Rühl and colleagues appears promising for further clinical development in an attempt to prevent EBV-associated malignancies.

\section{One step closer to an effective EBV vaccine}

Rühl and colleagues extensively and methodically investigated various combinations of homologous and heterologous vaccine sequences and resultant $\mathrm{T}$ cell responses for up to five months after vaccination, subsequently choosing the most potent heterologous prime-boost com- binations to further test in murine models (11). The decision to target EBNA1, as opposed to the other latency antigens, was logical, as EBNA1 is expressed on all $\mathrm{EBV}^{+}$ tumors, highlighting its potential role as a viral tumor antigen that can be targeted by both passive and active vaccination. Additionally, EBNA1 is consistently recognized by $\mathrm{CD} 4^{+} \mathrm{T}$ cells in virtually all healthy $\mathrm{EBV}$ carriers, further underscoring its key role as a candidate vaccine antigen (23).

In a quest to ensure enhanced delivery to both MHC class I and II pathways, Rühl et al. screened fusion proteins of EBNA1 and other dendritic cell- and myeloid subset-directed receptors. Ultimately, $\alpha$ DEC-E1 (a recombinant Ab targeting EBNA1 to DEC205 receptor in dendritic cells) or MVA with an invariant chain encoding EBNA1 (MVA-IiE1) as the optimal prime-boost regimen. These regimens effectively targeted EBNA1 to dendritic cells for efficient cross-presentation in combination with EBNA1-encoding adenovirus (Adeno-E1-LMP), which served to prime $\mathrm{CD} 8^{+} \mathrm{T}$ cells. This approach proved successful, effectively priming EBNA1specific $\mathrm{CD} 4^{+} \mathrm{T}$ cell responses and improving cross-presentation, which led to optimal $\mathrm{CD}^{+} \mathrm{T}$ cell responses and increased $\alpha$ EBNA1 IgG Ab titers. Overall, these studies confirm the central role of $\mathrm{CD}^{+}{ }^{+} \mathrm{T}$ cells in the priming and maintenance of $\mathrm{CD} 8^{+}$ $\mathrm{T}$ cells and establishing long-term postvaccination immune control of $\mathrm{EBV}$.

The availability of animal models to test EBV infection and tumor development is exceedingly limited. One of the inherent weaknesses of testing EBV vaccines in mouse models is the inability to evaluate whether sterilizing immunity and prevention of infection can be achieved, a long-standing challenge for EBV vaccine development. Nevertheless, the therapeutic and prophylactic heterologous vaccine approach used by Rühl and colleagues against mouse models of $\mathrm{T}$ cell and aggressive Burkitt-like lymphoma may provide some insight into preventative vaccine strategies against EBV-positive malignancies (11). Specifically, the authors demonstrated that prime-boost regimens ( $\alpha$ DEC-E1 plus AdenoE1-LMP or AdenoE1-LMP plus MVA-IiE1) resulted in complete tumor rejection of $\mathrm{T}$ cell lymphoma EL4 cells in 11 of 13 mice in a prophylactic vaccine challenge. Moreover, there was a substantial decrease in tumor burden, resulting in increased survival $(75 \%)$ of heterogeneously vaccinated mice. Likewise, in an EBNA1-induced B cell lymphoma model, a heterologous prime-boost prior to tumor injection decreased tumor burden, as measured by E1 DNA load, spleen histology, and E1-specific Western blotting, with $\alpha$ DEC-E1 plus Adeno-E1 and Adeno-E1-LMP plus MVA-IiE1 vaccine boosts. Ultimately, Rühl et al. provide convincing evidence that vaccination with two different prime-boost regimens can produce substantial $\mathrm{T}$ cell-mediated EBV immune control and $\mathrm{Ab}$ production and that efficacy of the vaccine and subsequent tumor control are dependent on a prime-boost regimen and subsequent stimulation of $\mathrm{CD}^{+}$and $\mathrm{CD} 8^{+} \mathrm{T}$ cells. For these reasons, these heterologous primeboost vaccines are indeed promising candidates for clinical application.

\section{The jury is still out}

One important caveat is that the specific EBNA1 epitope selected by Rühl et al. was modified by removal of the Gly-Ala repeats, rendering it specific for only highly immunogenic epitopes. Considering that many humans are infected with multiple strains of EBV, it remains to be seen whether the immune response reconstituted by this particular vaccine will provide immunogenicity against all strains of $E B V$ or will be limited to epitope-specific ones. Along the same lines, an EBNA1-predominant tumor was used in the syngeneic B cell lymphoma mouse model. Whether this EBNA1-specific vaccine will prove equally effective in $\mathrm{EBV}^{+}$malignancies that predominantly express other latency antigens, in addition to EBNA1, is an important question for further investigation. Last, the ideal timing of vaccine administration and its influence on the efficacy of EBNA1-primed $\mathrm{T}$ cells remain to be determined. Here, vaccination occurred within two weeks, either prior to or following tumor injection. One of the challenges of EBV vaccination in preventing development of EBV-associated malignancies is the prolonged latency period between EBV infection and tumor development. Evaluation of heterologous prime-boost vaccination in the prevention of EBV-associated malignancies at later time periods after vaccination will provide valuable information on the preventative effects of the vaccine. 


\section{Where do we go from here?}

The study by Rühl and colleagues demonstrates the potential application of primeboost heterologous vaccination in preventive and therapeutic strategies against EBV-associated malignancies. This strategy provides important feasibility data that may open the door to a generation of prime-boost heterologous vaccines targeting latent and lytic EBV genes, and thus various stages of the EBV life cycle. A better understanding of the gene expression profile in patients with EBV-related malignancies and correlation to EBV-specific $\mathrm{T}$ cell responses may provide insights into future vaccine targets. The approach can be extended to include vaccines targeting lytic and latent EBV antigens in an effort to enhance adaptive immune cell reconstitution and priming following vaccination. Specifically, further study of the latency period between vaccination and EBV infection and development of EBV-associated malignancy and the efficacy of repeated prime boosting over extended periods may shed light on further development of EBV vaccines.

As prevention of EBV infection has been the primary goal of EBV vaccine development, the promising data reported by Rühl et al. indicate a use for vaccination to prevent EBV-related malignancy, especially in regions of endemic EBV infection. The heterologous combination of Adeno-E1-LMP plus MVA-IiE1 (the vectors of which have been safely studied in clinical trials) (22) would probably easily translate into an early-phase trial. Furthermore, an exciting concept involves the combination of this promising vaccination strategy with adoptively transferred EBV-specific T lymphocytes, leading to augmented protection. Although we are still a long way from producing a vaccine able to prevent $\mathrm{EBV}$ infection, the strategy described by Rühl et al. highlights a potential strategy to prevent malignant transformation. A further understanding and identification of immune correlates associated with protection from $\mathrm{EBV}^{+}$malignancies will shed additional light on the effects of the vaccine on $\mathrm{T}$ cell priming and tumor control.

\section{Acknowledgments}

The authors' research efforts are funded in part by the Lymphoma Research Foundation (to RHR), the Lymphoma Specialized Program of Research Excellence (SPORE) (to RHR), the Leukemia and Lymphoma Society, Leukemia Texas (to RHR), the American Society of Hematology/Harold Amos Medical Faculty Development Program (ASH/AMFDP), the Amy Strelzer Manasevit/National Marrow Donor Program (NMDP), a Cancer Prevention and Research Institute of Texas (CPRIT) Training Fellowship (to SS), and an American Society of Gene and Cell Therapy (ASGCT) Career Development Award (to SS).

Address correspondence to: Rayne $\mathrm{H}$. Rouce, 1102 Bates Street, Suite 1770, Houston, Texas 77030, USA. Phone: 832.824.4723; Email: rhrouce@txch.org.

1. Rouce RH, Sharma S, Huynh M, Heslop HE. Recent advances in T-cell immunotherapy for haematological malignancies. Br J Haematol. 2017;176(5):688-704

2. Joura EA, et al. A 9-valent HPV vaccine against infection and intraepithelial neoplasia in women. N Engl J Med. 2015;372(8):711-723.

3. Paavonen J, et al. Efficacy of a prophylactic adjuvanted bivalent L1 virus-like-particle vaccine against infection with human papillomavirus types 16 and 18 in young women: an interim analysis of a phase III double-blind, randomised controlled trial. Lancet. 2007;369(9580):2161-2170.

4. Chang $\mathrm{MH}$, et al. Universal hepatitis B vaccination in Taiwan and the incidence of hepatocellular carcinoma in children. Taiwan Childhood Hepatoma Study Group. N Engl J Med. 1997;336(26):1855-1859.

5. Dubois MF, Pourcel C, Rousset S, Chany C, Tiollais P. Excretion of hepatitis B surface antigen particles from mouse cells transformed with cloned viral DNA. Proc Natl Acad Sci U S A. 1980;77(8):4549-4553.

6. Moutschen M, et al. Phase I/II studies to evaluate safety and immunogenicity of a recombinant gp350 Epstein-Barr virus vaccine in healthy adults. Vaccine. 2007;25(24):4697-4705.

7. Sokal EM, et al. Recombinant gp350 vaccine for infectious mononucleosis: a phase 2, randomized, double-blind, placebo-controlled trial to evaluate the safety, immunogenicity, and efficacy of an Epstein-Barr virus vaccine in healthy young adults. JInfect Dis. 2007;196(12):1749-1753.

8. Rees L, et al. A phase I trial of Epstein-Barr virus gp350 vaccine for children with chronic kidney disease awaiting transplantation. Transplantation. 2009;88(8):1025-1029.
9. Cohen JI, Fauci AS, Varmus H, Nabel GJ. Epstein-Barr virus: an important vaccine target for cancer prevention. Sci Transl Med. 2011;3(107):107fs7.

10. Cohen JI, Mocarski ES, Raab-Traub N, Corey L, Nabel GJ. The need and challenges for development of an Epstein-Barr virus vaccine. Vaccine. 2013;31(suppl 2):B194-B196.

11. Rühl J, et al. Heterologous prime-boost vaccination protects against EBV antigen-expressing lymphomas. J Clin Invest. 2019;129(5):2071-2087.

12. Yao QY, et al. Frequency of multiple Epstein-Barr virus infections in T-cell-immunocompromised individuals. J Virol. 1996;70(8):4884-4894.

13. Roskrow MA, et al. Epstein-Barr virus (EBV)-specific cytotoxic $\mathrm{T}$ lymphocytes for the treatment of patients with EBV-positive relapsed Hodgkin's disease. Blood.1998;91(8):2925-2934.

14. Rooney CM, et al. Use of gene-modified virus-specific T lymphocytes to control Epstein-Barr-virus-related lymphoproliferation. Lancet. 1995;345(8941):9-13.

15. Bollard CM, et al. Sustained complete responses in patients with lymphoma receiving autologous cytotoxic T lymphocytes targeting Epstein-Barr virus latent membrane proteins. J Clin Oncol. 2014;32(8):798-808.

16. Gu SY, et al. First EBV vaccine trial in humans using recombinant vaccinia virus expressing the major membrane antigen. Dev Biol Stand. 1995;84:171-177.

17. Elliott SL, et al. Phase I trial of a CD8 ${ }^{+} \mathrm{T}$-cell peptide epitope-based vaccine for infectious mononucleosis. J Virol. 2008;82(3):1448-1457.

18. Lin CL, et al. Immunization with Epstein-Barr Virus (EBV) peptide-pulsed dendritic cells induces functional $\mathrm{CD}^{+} \mathrm{T}$-cell immunity and may lead to tumor regression in patients with EBV-positive nasopharyngeal carcinoma. Cancer Res. 2002;62(23):6952-6958.

19. Hui EP, et al. Phase I trial of recombinant modified vaccinia ankara encoding Epstein-Barr viral tumor antigens in nasopharyngeal carcinoma patients. Cancer Res. 2013;73(6):1676-1688.

20. Taylor GS, et al. A recombinant modified vaccinia ankara vaccine encoding Epstein-Barr Virus (EBV) target antigens: a phase I trial in UK patients with EBV-positive cancer. Clin Cancer Res. 2014;20(19):5009-5022.

21. Chia WK, et al. A phase II study evaluating the safety and efficacy of an adenovirus- $\triangle$ LMP1-LMP2 transduced dendritic cell vaccine in patients with advanced metastatic nasopharyngeal carcinoma. Ann Oncol. 2012;23(4):997-1005.

22. Ogwang C, et al. Prime-boost vaccination with chimpanzee adenovirus and modified vaccinia Ankara encoding TRAP provides partial protection against Plasmodium falciparum infection in Kenyan adults. Sci Transl Med. 2015;7(286):286re5.

23. Münz C, et al. Human CD4(+) T lymphocytes consistently respond to the latent EpsteinBarr virus nuclear antigen EBNA1. J Exp Med. 2000;191(10):1649-1660. 